2-3 years) at birth with three doses of recombinant hepatitis $B$ vaccine. Tests revealed that 8 participants had evidence of new hepatitis $B$ infection (their sera tested positive for antibody to HBV core antigen), but none were chronically infected. Among the 97 participants who had no evidence of HBV infection, only 7 had protective serum titers $(\geq 10 \mathrm{mlU} / \mathrm{ml})$ of antibodies to hepatitis B surface antigen. Furthermore, 2 weeks after 96 of the 97 individuals received a vaccine booster, only 46 (47.9\%) displayed an anamnestic response (a fourfold increase in antibodies to hepatitis $B$ surface antigen titers, with peak $\geq 10 \mathrm{mlU} / \mathrm{ml}$ ).

The authors note that absence of an anamnestic response might indicate waning immunity. Should further research indicate the need for an additional dose of HBV vaccine during childhood, its administration at 4-5 years of age would be best, as this timing would coordinate with other recommended vaccinations and maximize the likelihood of response.

Original article Bialek SR et al. (2008) Persistence of protection against hepatitis $B$ virus infection among adolescents vaccinated with recombinant hepatitis $B$ vaccine beginning at birth: a 15-year follow-up study. Pediatr Infect Dis J 27: 881-885

\section{High iron intake and body iron stores are not risk factors for Barrett's esophagus}

Barrett's esophagus is associated with a 30-40-fold increase in risk for esophageal adenocarcinoma. Animal studies suggest that high iron intake and elevated body iron stores enhance risk of both Barrett's esophagus and esophageal adenocarcinoma.

Corley et al. conducted a case-control study among 3.3 million members of Kaiser Permanente in Northern California. Data were collected on 319 individuals with Barrett's esophagus, 312 patients with GERD, which is a risk factor for Barrett's esophagus, and 313 population controls. Risk of Barrett's esophagus was significantly lower in individuals whose dietary iron intake was in the highest quartile than in those whose intake was in the lowest quartile (a $63 \%$ decrease in risk versus that of controls, and a $58 \%$ decrease versus patients with GERD). Risk was not significantly associated with total iron intake (dietary and supplements). Risk of Barrett's esophagus was also significantly reduced in individuals in the fourth quartile versus the first of body iron stores, as measured by their serum ferritin levels (a $76 \%$ decrease in risk versus controls, and of $40 \%$ versus patients with GERD) and by transferrin saturation (a 34\% decrease in risk versus controls only).

Antioxidants may limit iron-related oxidative damage, but the authors found no evidence of a confounding effect of antioxidant intake on their findings. Consequently, high iron levels do not seem to be a risk factor for Barrett's esophagus; in fact, they might have a protective effect.

Original article Corley AD et al. (2008) Iron intake and body iron stores as risk factors for Barrett's esophagus: a community-based study. Am J Gastroenterol [doi:10.1111/ j.1572-0241.2008.02156.x 